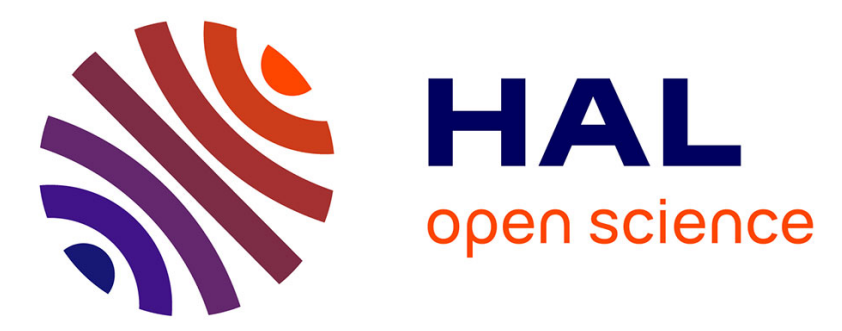

\title{
Open-source, high-throughput ultrasound treatment chamber
}

Torstein Yddal, Sandy Cochran, Odd Helge Gilja, Michiel Postema, Spiros Kotopoulis

\section{- To cite this version:}

Torstein Yddal, Sandy Cochran, Odd Helge Gilja, Michiel Postema, Spiros Kotopoulis. Open-source, high-throughput ultrasound treatment chamber. Biomedical Engineering = Biomedizinische Technik, 2015, 60 (1), pp.77-87. 10.1515/bmt-2014-0046 . hal-03192888

\section{HAL Id: hal-03192888 \\ https://hal.science/hal-03192888}

Submitted on 12 Apr 2021

HAL is a multi-disciplinary open access archive for the deposit and dissemination of scientific research documents, whether they are published or not. The documents may come from teaching and research institutions in France or abroad, or from public or private research centers.
L'archive ouverte pluridisciplinaire HAL, est destinée au dépôt et à la diffusion de documents scientifiques de niveau recherche, publiés ou non, émanant des établissements d'enseignement et de recherche français ou étrangers, des laboratoires publics ou privés. 


\section{Torstein Yddal, Sandy Cochran, Odd Helge Gilja, Michiel Postema and Spiros Kotopoulis* Open-source, high-throughput ultrasound treatment chamber}

\begin{abstract}
Studying the effects of ultrasound on biological cells requires extensive knowledge of both the physical ultrasound and cellular biology. Translating knowledge between these fields can be complicated and time consuming. With the vast range of ultrasonic equipment available, nearly every research group uses different or unique devices. Hence, recreating the experimental conditions and results may be expensive or difficult. For this reason, we have developed devices to combat the common problems seen in state-of-the-art biomedical ultrasound research. In this paper, we present the design, fabrication, and characterisation of an open-source device that is easy to manufacture, allows for parallel sample sonication, and is highly reproducible, with complete acoustic calibration. This device is designed to act as a template for sample sonication experiments. We demonstrate the fabrication technique for devices designed to sonicate 24-well plates and OptiCell ${ }^{\mathrm{TM}}$ using three-dimensional (3D) printing and low-cost consumables. We increased the pressure output by electrical impedance matching of the transducers using transmission line transformers, resulting in an increase by a factor of 3.15. The devices cost approximately $€ 220$ in consumables, with a major portion attributed to the 3D printing, and can be fabricated in approximately 8 working hours. Our results show that, if our protocol is followed, the mean acoustic output between devices has a variance of $<1 \%$. We openly provide the $3 \mathrm{D}$ files and
\end{abstract}

\footnotetext{
*Corresponding author: Spiros Kotopoulis, National Centre for Ultrasound in Gastroenterology, Haukeland University Hospital, Jonas Lies vei 65, 5021 Bergen, Norway, E-mail: Spiros.Kotopoulis@ helse-bergen.no; and Department of Physics and Technology, University of Bergen, Allégaten 55, 5007 Bergen, Norway Torstein Yddal: National Centre for Ultrasound in Gastroenterology, Haukeland University Hosptial, Jonas Lies vei 65, 5021 Bergen, Norway; and Department of Physics and Technology, University of Bergen, Allégaten 55, 5007 Bergen, Norway

Sandy Cochran: Institute for Medical Science and Technology, University of Dundee, Wilson House, 1 Wurzburg Loan, Dundee DD2 1FD, UK
}

Odd Helge Gilja: National Centre for Ultrasound in Gastroenterology, Haukeland University Hosptial, Jonas Lies vei 65, 5021 Bergen, Norway; and Department of Clinical Medicine, University of Bergen, Jonas Lies vei 65, 5021 Bergen, Norway

Michiel Postema: Department of Physics and Technology, University of Bergen, Allégaten 55, 5007 Bergen, Norway operation software allowing any laboratory to fabricate and use these devices at minimal cost and without substantial prior know-how.

Keywords: Sonoporation; experimentation devices; rapid prototyping; ultrasound transducers.

DOI 10.1515/bmt-2014-0046

Received May 19, 2014; accepted October 6, 2014; online first November 4, 2014

\section{Introduction}

Ultrasound is well known for its diagnostic uses, for example, in foetal scanning and industrial non-destructive testing. Nevertheless, it is also used in a further vast range of research fields, such as chemistry, oil and gas prospecting, nanotechnology, and aquatic zoology [2, 19, 20, 26]. Ultrasound is also commonly used in modern therapy, for example, in physiotherapy [9] to heat deep tissue and disrupt kidney stones [5]. More recent developments in therapeutic ultrasound are focused ultrasound surgery [15] and ultrasound-mediated drug and gene delivery $[3,13,23]$.

Studying the effects of ultrasound on biological cells requires extensive knowledge of both the physical ultrasound parameters and cellular biology. To date, a majority of biology- and physics-related research, especially in in vitro studies, has a paucity of content in either one of the fields, making it difficult to recreate the experimental conditions and subsequently the results. Although there are several commercial devices available (such as the Sonidel SP100 [17] or Sonitron 2000 [11]), they all require re-calibration and there is limited control on the acoustic conditions. Some solutions use extremely expensive devices, such as 1000-element three-dimensional (3D) phased arrays [6], treat a single well at a time, contaminating the ultrasound transducer [14] and have low throughput [7], are not open-source to allow system replication [18], or in certain cases, work in the near field of the ultrasound transducer and simply do not calibrate the sound field [16]. Having a readily available opensource device will open the field of ultrasound-mediated 
research to many more laboratories that could currently not perform such research due to either cost or complexity. Hopefully, more researchers in the field of ultrasoundmediated therapy will help further our understanding and the possibilities of such a therapeutic modality. For these reasons, we have developed devices to combat the common problems seen in state-of-the-art biomedical ultrasound research, helping produce a tool that is reliable, reproducible, and can help reduce the complexity during experimental and common laboratory errors.

These devices have to be easy to use by someone with limited or no knowledge of ultrasonics, compact enough to fit in a sterile environment, low cost, able to work with existing biological hardware products, e.g., 24-well plates or OptiCells ${ }^{\mathrm{TM}}$, allow high throughput, yet easy to modify for mounting the different apparatus such as Eppendorf tubes or Petri dishes. In addition, it is important that these devices can be manufactured easily in a laboratory with basic equipment, without the need of extensive skills and knowledge, and that the results are repeatable, notwithstanding the small inherent variations of custom manufacturing.
Ideally, these devices should be open-source, allowing anyone to fabricate, modify, and use them.

This paper describes an open-source ultrasound treatment chamber for parallel sonication of six samples, compliant with a vast range ultrasound driving equipment, i.e., waveform generators and amplifiers, using a simple, "one-button" interface.

\section{Material and methods}

\section{Design}

Figure 1 shows a computer-aided design (CAD) of the two devices designed for cell sample sonication. Both devices were designed with six ultrasound transducers, allowing simultaneous treatment of six samples. Both devices are based on the same template.

Device A was designed for acoustic treatment of both floating cells and adherent cells. It is based on a 24-well plate. This plate was chosen because it allows sufficiently large samples to be treated and subsequently physically separated for evaluation. It also ensures that there is no mixing between samples, so that different test conditions can be evaluated simultaneously. In this device, the transducers are

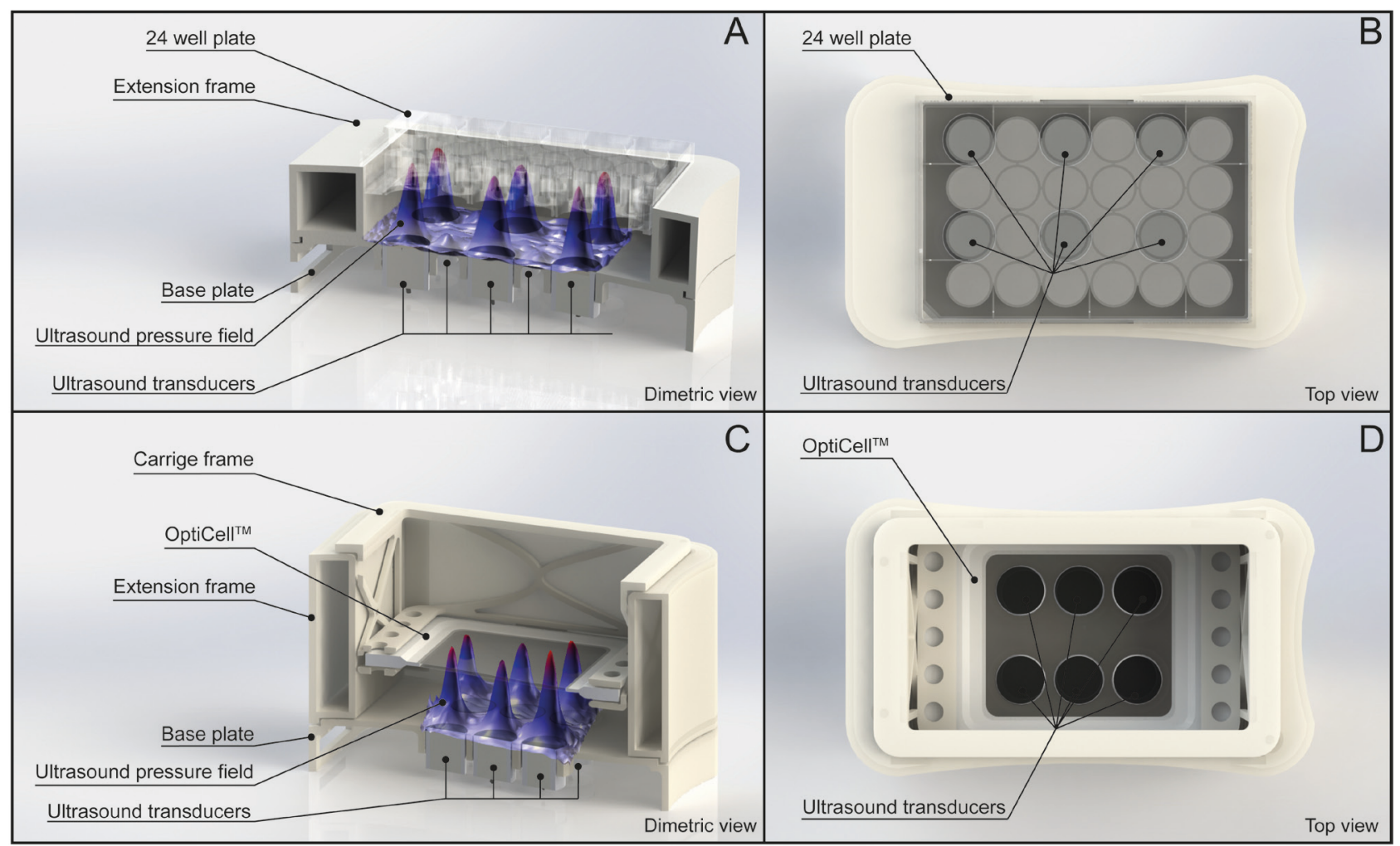

Figure 1 Computer generated renderings of prototype devices. Cutaway diagram showing assembly of ultrasound sonicators for 24-well plates ( $A$ and $B$ ) and OptiCells ${ }^{T M}(C$ and $D)$ including the acoustic pressure fields. (B and D) Top view and position of the ultrasound transducers for both devices. In the OptiCell ${ }^{T M}$ sonicator, the ultrasound transducers have been places substantially closer. 
arranged in such a way that their acoustic foci are aligned with the vertical centres of the plates (Figure 1B). The height of the extension frame ensures the acoustic focus is exactly in the centre of the plate. The device is designed to place the plate inverted. The top of the plate is covered with a self-adhering thin membrane (TopSeal ${ }^{\mathrm{TM}}$-A; Perkin Elmer, Waltham, MA, USA) measured to have $<1 \%$ pressure attentuation at $1 \mathrm{MHz}$. By positioning the 24-well plate upright, adherent cells can be used, as the acoustic foci will align with the base of the plate. In such cases co-treatment experiments can be performed with different chemicals in each well with the same ultrasound in each well. Device B was designed for the treatment of adherent cells and is based on an OptiCell ${ }^{\mathrm{TM}}$. An OptiCell ${ }^{\mathrm{TM}}$ was chosen for its versatility and acoustic compliance. OptiCells ${ }^{\mathrm{TM}}$ are $10-\mathrm{ml}$ chambers, where the upper and lower walls are made of $<100-\mu \mathrm{m}$-thick polystyrene membranes, resulting in negligible acoustic attenuation when submerged and filled with water [4]. Using an OptiCell ${ }^{\mathrm{TM}}$, the same cell culture can be treated simultaneously and then evaluated using any desired method. Furthermore, after treatment, the membrane can be cut, allowing samples to be evaluated separately.

In device $\mathrm{B}$, the transducers were placed closer together than in device A (Figure 1D) so as the acoustic focus lies within the membrane area, with minimal acoustic interference. Here, the extension frame was substantially longer allowing acoustic propagation into the far field. A carriage frame was used to lower and accurately align the OptiCell ${ }^{\mathrm{TM}}$ in the acoustic focus.

The distance of the acoustic focus $(z)$ was determined by [21]

$$
z=\frac{D^{2} f}{4 c},
$$

where $D$ is the diameter of the piezoelectric element, $f$ is the centre frequency transmitted, and $c$ is the speed of sound in the propagation medium.

\section{Frame fabrication}

The plastic frames were fabricated using a ProJet 1500 3D printer (3D Systems, Rock Hill, SC, USA). To ensure a correct fit, test pieces were printed to determine the accuracy of the 3D printer. It was determined that holes were printed $0.3 \mathrm{~mm}$ smaller, and extrusions were printed $0.2 \mathrm{~mm}$ larger than designed. These tolerances were then imported into the 3D model, and an additional $0.5 \mathrm{~mm}$ was added between components, resulting in smooth non-interfering assembly. When exporting the 3D design as and STL file for printing, it was essential that the resolution of the file was set to $0.01 \mathrm{~mm}$ or less in deviation and $5^{\circ}$ or less in angle. This was necessary to ensure all boundaries were printed at the highest resolution and accuracy.

Each component required $5-8 \mathrm{~h}$ to print. Initial setup takes approximately 15-30 min to check the correct orientation and support location. It was found to be paramount to orientate the component correctly so the printing support structures were not placed on critical surfaces, e.g., transducer holes or inner walls. Once the 3D print was complete, $30 \mathrm{~min}$ of post-processing, cleaning, and final curing of the frame components was required. A further 30 min was required to safely break the supports away and sand the surface. Sanding was performed in two stages: stage 1, dry sanding with a mechanical sander (MS800B Mouse; Black \& Decker, New Britain, CT, USA), and stage 2, wet sanding by hand using P180 grit sandpaper (3M, St. Paul, MN, USA).
Both devices were designed to allow all transducers to be driven either simultaneously or independently via a simple connector. For this reason, a DB-25 connector was used for the transducer interface. The female connector was attached to the device. All transducers were independently connected but with a common earth. Within the casing of the male connector, all six transducers could be interconnected, or six independent cables could be used. This allows for maximum connection and transducer driving flexibility.

The frames can be manufactured using any process, whether 3D printing or computer numerical control (CNC) machining, as long as the material is watertight and water-resistant.

All devices were designed in SolidWorks 2013 (Dassault Systèmes SolidWorks, Waltham, MA, USA).

\section{Ultrasound transducers}

The ultrasound transducers were based on $\varnothing 15$-mm, 2-mm thick, PZ26 Navy Type I, lead zirconate titanate (PZT) discs, with screen-printed silver electrodes (Meggit PLC, Christchurch, UK). For the transducer cases, 17-mm-inner diameter (ID), 25-mm-outer diameter (OD), 25-mm-long cylinders were used. The piezoelectric elements were connected to the inner core of RG178/U coaxial cable, and the cases were connected to the shielding using conductive silver-loaded epoxy (Oxford Instruments, Abingdon, UK). Backing and encasing material consisted of K1 glass bubbles (3M) mixed with EpoFix epoxy (Struers, Ballerup, Denmark) at a w/w ratio of 8-9:1, respectively. At this ratio, the glass bubble/epoxy mixture was still thin enough to pour into the transducer and fill small gaps. Increasing the $\mathrm{w} / \mathrm{w}$ ratio results in a thick paste, requiring the backing to be forced into place, typically dislodging cables or damaging elements. Our ratio results in a backing with density of $0.6 \mathrm{~g} / \mathrm{cm}^{3}$. Once the backing was poured into the transducer case, the transducers were placed in a vacuum chamber and were vacuum cycled 20 times between 0.5 mbar and atmospheric pressure every $20 \mathrm{~s}$, removing any trapped air pockets and ensuring that the transducers were homogeneously held in place. Conductive paint was used to connect the front electrode surface to the case. The assembly protocol of the transducers has been thoroughly described previously [12]. Figure 2 shows a cutaway of an assembled ultrasound transducer assembled with this protocol. The ultrasound transducers

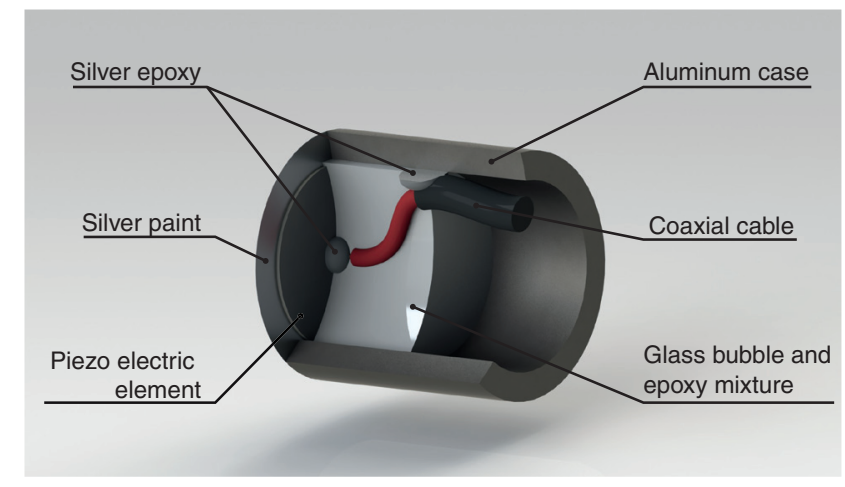

Figure 2 Cutaway diagram showing components and assembly of the ultrasound transducers. The front face of the transducer has been connected to the earth via the case. This ensures a smooth front surface with no electrodes. A low-density glass bubble and epoxy mixture has been used as backing to support the piezo element with minimal damping. 
were bonded to the frame using EpoFix epoxy (Struers A/S). Transducers can be interchangeable using polytetrafluoroethylene (PTFE) tape to seal the transducers in the base plate.

\section{Electrical impedance matching}

For maximum efficiency, the electrical impedance of the ultrasound was checked and matched to the characteristic impedance of the driving source, i.e., $50 \Omega$ in the present work. The electrical impedance was measured using a TE1000 (TrewMac, Adelaide, Australia). Impedance is typically measured in the Cartesian form with real and imaginary components, i.e.,

$$
Z=R+j X,
$$

where $R$ is the real impedance and $j X$ is the imaginary impedance. The amount of electrical reflection $(\Gamma)$ is calculated by

$$
\Gamma=\frac{Z-Z_{0}}{Z+Z_{0}},
$$

where $Z$ is the transducer impedance and $Z_{0}$ is the characteristic impedance, typically $50 \Omega$. Hence, the reflection can be calculated by combing Eqs. 2 and 3, grouping the real and imaginary parts, and conjugating the result to give

$$
\Gamma=\frac{R^{2}-Z_{0}^{2}+X^{2}}{\left(R+Z_{0}\right)^{2}+X^{2}}+j \frac{2 Z_{0} X}{\left(R+Z_{0}\right)^{2}+X^{2}} .
$$

The magnitude of the reflection coefficient $(\delta)$ in polar form indicates the amount of electrical reflection from the transducer as a fraction and can be calculated with

$$
\delta=\sqrt{\left(\frac{R^{2}-Z_{0}^{2}+X^{2}}{\left(R+Z_{0}\right)^{2}+X^{2}}\right)^{2}+\left(\frac{2 Z_{0} X}{\left(R+Z_{0}\right)^{2}+X^{2}}\right)^{2}} .
$$

Hence, $\delta=1$ indicates $100 \%$ reflection and $\delta=0$ indicates $0 \%$ reflection and thus perfect impedance matching.

To match the ultrasound transducers, unbalanced transmission line transformers were used as these have very low losses, 0.02-0.04 dB. The flux is effectively cancelled out in these transformers, minimising heating during operation. Because they require only a few turns, they are extremely easy to construct [25].

The transmission line transformers were based on 12.5-mm-OD, 8.0-mm-ID, 6.5-mm-tall ferrite toroids (Amidon Associates, Costa Mesa, CA, USA) and 28-AWG, 7-core wire with $250-\mu \mathrm{m}$ insulation.

\section{Acoustic field characterisation}

The ultrasound fields generated by these two devices were calibrated using a custom-made, automated, and closed-loop 3D scanning system. The scanning system had an accuracy of $30 \mu \mathrm{m}$ with a repeatability of $<2.5 \mu \mathrm{m}$ and with a resolution of $0.4 \mu \mathrm{m}$. A calibrated $200-\mu \mathrm{m}$ polyvinylidene fluoride (PVDF) needle hydrophone (Precision Acoustics, Dorset, UK) was used to measure the acoustic pressures. The axial propagation direction was denoted as the $z$-axis, and the lateral and elevation directions were denoted as $x$ and $y$, respectively. During the calibration process, all elements in the device were driven simultaneously using a five-cycle tone burst generated by an AFG3002 function generator (Tektronix, Beaverton, OR, USA) connected to a 2100L, 100-W, RF power amplifier (Electronics \& Innovation, Rochester, NY, USA) via a -20-dB attenuator to simulate true working conditions. The devices were calibrated in a free field, i.e., only the base plate, without the OptiCell ${ }^{\mathrm{TM}}$ or 24-well plates in place. The embedded RMS power meter in the RF amplifier was used to measure the forward and reflected powers. The results from the RMS power meter were also used to measure the effectiveness of the impedance matching.

The centre frequency was determined by placing the transducer at a planar target and excited using a tone burst over a range of frequencies. The ratio of the transmitted to received signal determined the sensitivity. The centre frequency, $f_{c}$, was determined by

$$
f_{\mathrm{c}}=f_{1}+\left(\frac{f_{\mathrm{u}}-f_{1}}{2}\right)
$$

where $f_{\mathrm{u}}$ is the upper frequency limit and $f_{1}$ is the lower frequency limit. The upper and lower frequency limits were determined by the corresponding value $-3 \mathrm{~dB}$ from maximum sensitivity. The transducer bandwidth, $B W$, percentage bandwidth, $\% B W$, and mechanical quality factor, $Q_{\mathrm{m}}$, were calculated by

$$
\begin{gathered}
B W=f_{\mathrm{u}}-f_{1}, \\
\% B W=100 \frac{B W}{f_{\mathrm{c}}}, \\
Q_{\mathrm{m}}=\frac{f_{\mathrm{c}}}{B W} .
\end{gathered}
$$

The mechanical quality factor indicates the degree of damping in the transducer. A high $Q_{\mathrm{m}}$ typically indicates higher acoustic output. A low $Q_{\mathrm{m}}$ indicates high internal damping, hence broader bandwidth and less ringing [21].

Nonlinear propagation is a critical phenomenon that can affect the maximum output of an ultrasound transducer at a desired frequency. As the nonlinearity increases with the acoustic pressure and harmonics are formed, the acoustic energy gets transferred to these higher harmonics. As attenuation is substantially greater at higher frequencies, there is a maximum limit to the acoustic pressure. As the transient wave propagates, the energy is transferred to the positive components of the waveforms. The more nonlinear the acoustic wave, the larger the difference [22]. Hence, the nonlinearity ratio, $N L R$, was evaluated by

$$
N L R=\left|\frac{P_{\text {peak-positive }}}{P_{\text {peak-negative }}}+1\right|,
$$

where $P_{\text {peak-positive }}$ is the peak-positive pressure and $P_{\text {peaknegative }}$ is the peak-negative acoustic pressure. The higher the $N L R$, the more nonlinear the acoustic propagation. All measurements were performed in distilled, deionised, and degassed water and calibrated based on the ASTM and International Electrotechnical Commission guidelines $[1,8]$.

\section{Statistics}

All statistics were performed using Prism v5.0a (GraphPad Software, La Jolla, CA, USA). An unpaired Student's t-test was used to compare the outputs of the devices. The results were considered statistically significant when $\mathrm{p} \leq 0.05$. 


\section{Results}

\section{Electrical impedance matching}

At the primary resonance, the mean impedance of the transducers was $109( \pm 13) \Omega+j 50( \pm 11) \Omega$ at $1.01 \mathrm{MHz}$ and $76( \pm 24) \Omega+j 50( \pm 21) \Omega$ at $0.96 \mathrm{MHz}$ for devices $\mathrm{A}$ and $\mathrm{B}$, respectively. Hence, for optimum electrical matching to $50 \Omega$, a transformer with an impedance ratio of 2.25:1 was required. Figure $3 \mathrm{~A}$ and $\mathrm{B}$ shows the schematic of two types of transmission line transformers used for this application. Both transformers were trifillar, with seven turns.
Transformer A was based on the W2FMI-2.25:1-HU50, while transformer B was based on the W2FMI-2.25:1-HU112.5 [24] Figure $3 \mathrm{C}$ shows the wiring diagram for independently connecting all transducers.

Figure 4 shows the effect on $\delta$ of impedance matching using transmission line transformers. In device A, prior to impedance matching, the reflection coefficient was 0.45 ; after impedance matching, this dropped 0.19. Combining all six transducers together showed a further drop to 0.09 , but a slight frequency shift to $1.08 \mathrm{MHz}$ was also observed.

In device $\mathrm{B}$, prior to impedance matching, the reflection coefficient was 0.46. After impedance matching, this

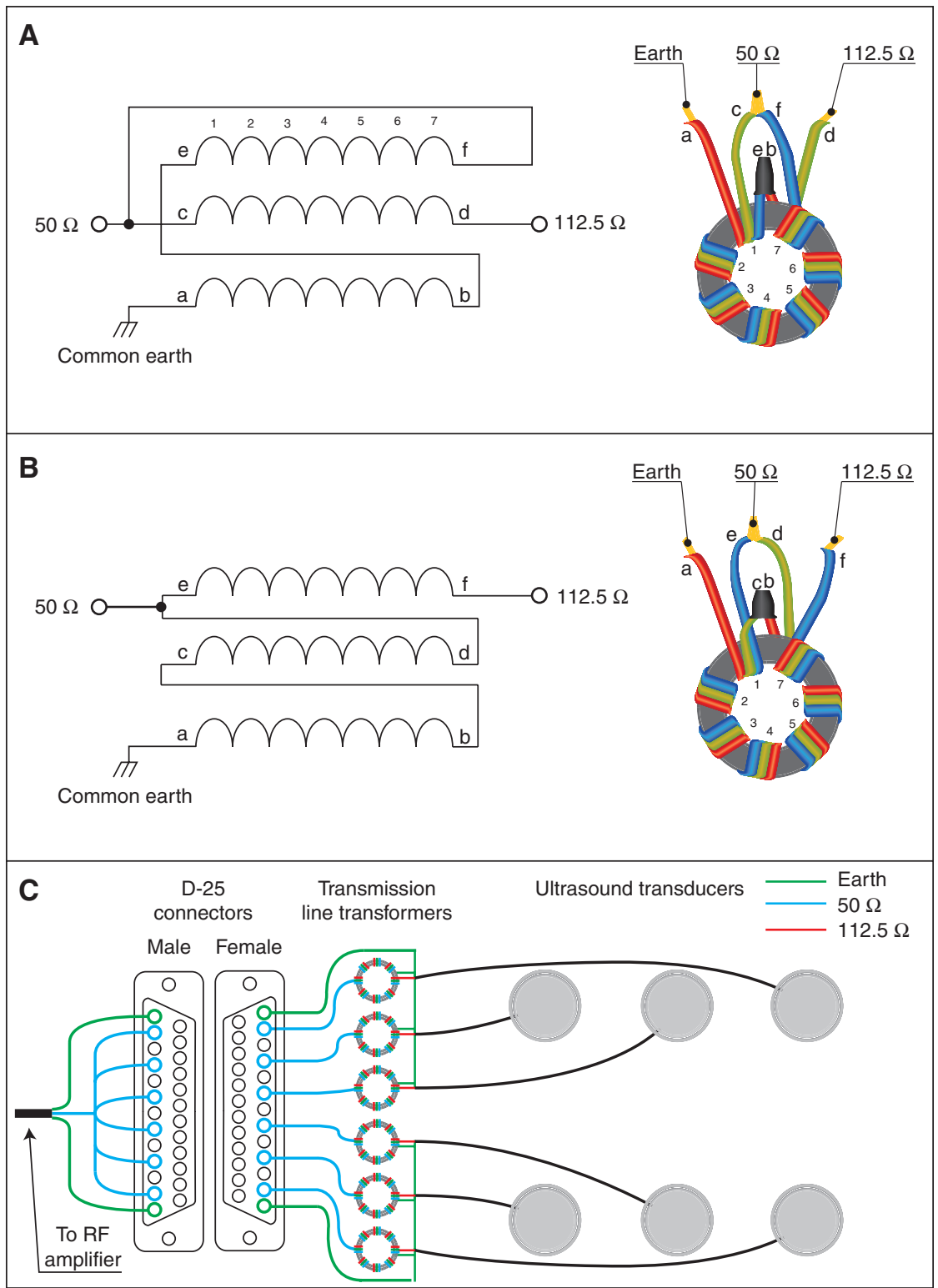

Figure 3 Electrical impedance matching and connection diagrams. Transmission line transformers used to match $112.5-\Omega$ transducers to $50 \Omega$ (A and B). Both variation result in the same impedance ratio and can be used interchangeably. Wiring schematic from transducers to amplifier (C) showing the independent connection of each transducer in the case. All transducers can be driven simultaneously via an interconnected male connector. 

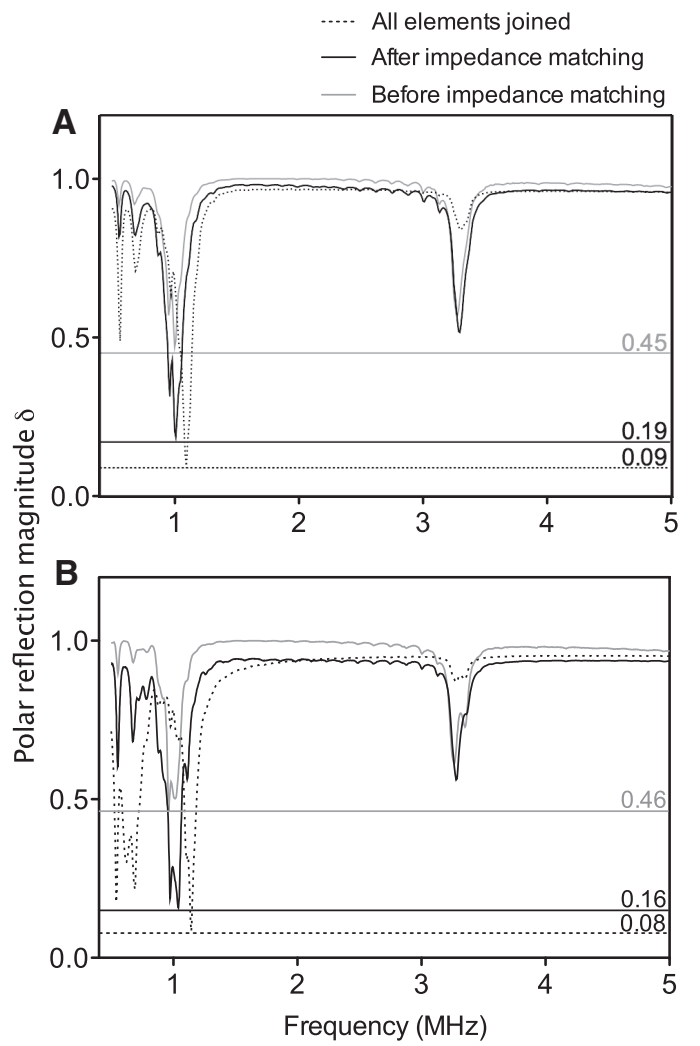

Figure 4 Electrical reflection coefficient as a function of frequency for both devices, with and without impedance matching. Mean polar reflection magnitude $(\delta)$ as a function of frequency for the 24-well plate (A) and the OptiCell ${ }^{\mathrm{TM}}$ sonicator (B). A minimum reflection can be seen at the centre frequencies and reduced reflections at the equivalent third harmonics. After impedance matching, the reflection coefficient is significantly reduced at the centre frequency but not at the third harmonic. Connecting all elements in a device together reduces the reflection coefficient further more.

dropped to 0.16. Combining all six transducers showed a further drop to 0.08 , along with a slight frequency shift to 1.14 MHz. A low reflection magnitude was also observed between 0.6 and $0.8 \mathrm{MHz}$.

\section{Acoustic field characterisation}

Figure 5 shows the normalised frequency response of the two devices from $400 \mathrm{kHz}$ to $4 \mathrm{MHz}$. A small shift $(+80 \mathrm{kHz})$ of the peak sensitivity of device B can be seen. Nevertheless, the bandwidth for both devices is identical at $0.17 \mathrm{MHz}$, as shown in Table 1. The OptiCell ${ }^{\mathrm{TM}}$ sonicator also showed an increased sensitivity between 0.6 and $0.8 \mathrm{MHz}$. The frequency response directly correlated with the impedance measurements.

Two-dimensional scans of the sonicators can be seen in Figure 6. Scan A shows the $x y$ scan of device B at the acoustic

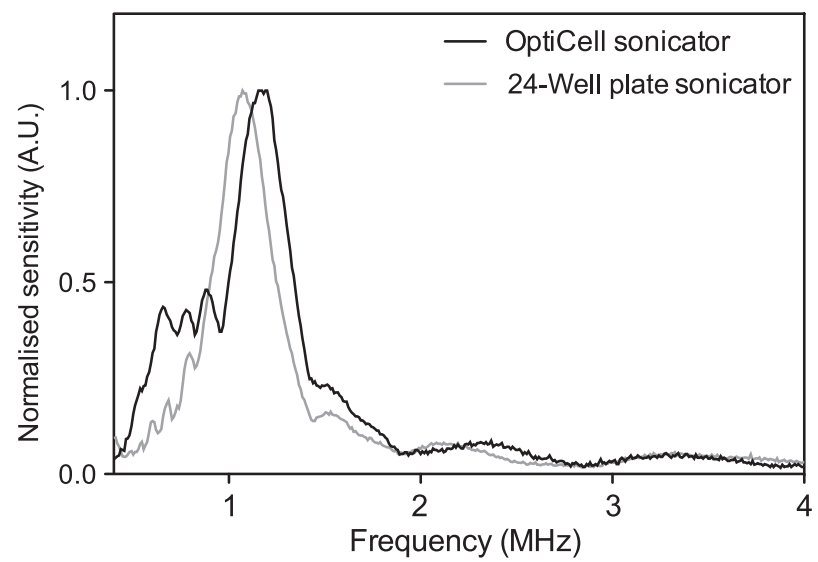

Figure 5 Frequency sensitivity of sonicators. Peak sensitivity can be seen at 1.08 and $1.17 \mathrm{MHz}$ for the 24-well plate and OptiCell ${ }^{\mathrm{TM}}$ sonicator, respectively. Increased sensitivity is evident between 0.6 and $0.8 \mathrm{MHz}$ in the OptiCell ${ }^{\mathrm{TM}}$ sonicator. This directly correlates to the reflection coefficient magnitude shown in Figure 4.

focus; the position is shown by line A-A in scan B. Scan B shows the $x z$ scan of the two central transducers from device $\mathrm{B}$; the location is shown by line B-B in scan A. Scan C shows the $x y$ scan of the device $A$ at the acoustic focus.

Owing to the close proximity of the transducers in device $\mathrm{B}$, minor constructive interference can be seen (Figure 6A). These side-lobes were $24 \mathrm{~dB}$ less than the peak pressure. No interference was seen in device A.

Following Eq. 1, the acoustic focal distances of devices A and B should be 41 and $44 \mathrm{~mm}$, respectively. The 3D scans showed the peak values at 39 and $42 \mathrm{~mm}$, respectively. Using a-2-dB cutoff, the acoustic focal length was $43 \pm 3 \mathrm{~mm}$; hence, the difference in acoustic pressure between the theoretical and the measured acoustic focus was negligible at $<0.2 \mathrm{~dB}$.

Using a -3-dB cutoff, the focal area was significantly different between the two devices $(p=0.0013)$. Figure 7A

Table 1 Frequency response of sonicators using a -3-dB cutoff.

\begin{tabular}{lrr}
\hline & $\begin{array}{r}\text { 24-Well plate } \\
\text { sonicator }\end{array}$ & $\begin{array}{r}\text { OptiCell } \\
\text { sonicator }\end{array}$ \\
\hline Peak sensitivity $(\mathrm{MHz})$ & 1.07 & 1.15 \\
$f_{1}(\mathrm{MHz})$ & 0.90 & 1.00 \\
$f_{\mathrm{c}}(\mathrm{MHz})$ & 1.08 & 1.17 \\
$f_{\mathrm{u}}(\mathrm{MHz})$ & 1.25 & 1.34 \\
$B W(\mathrm{MHz})$ & 0.17 & 0.17 \\
$Q_{\mathrm{m}}$ & 6.32 & 6.86 \\
$\% B W(\mathrm{MHz})$ & 15.82 & 14.57 \\
\hline
\end{tabular}

A small centre frequency shift of $90 \mathrm{kHz}$ between devices is evident, yet both devices have an identical bandwidth of $170 \mathrm{kHz}$ and a similar mechanical quality factor. 

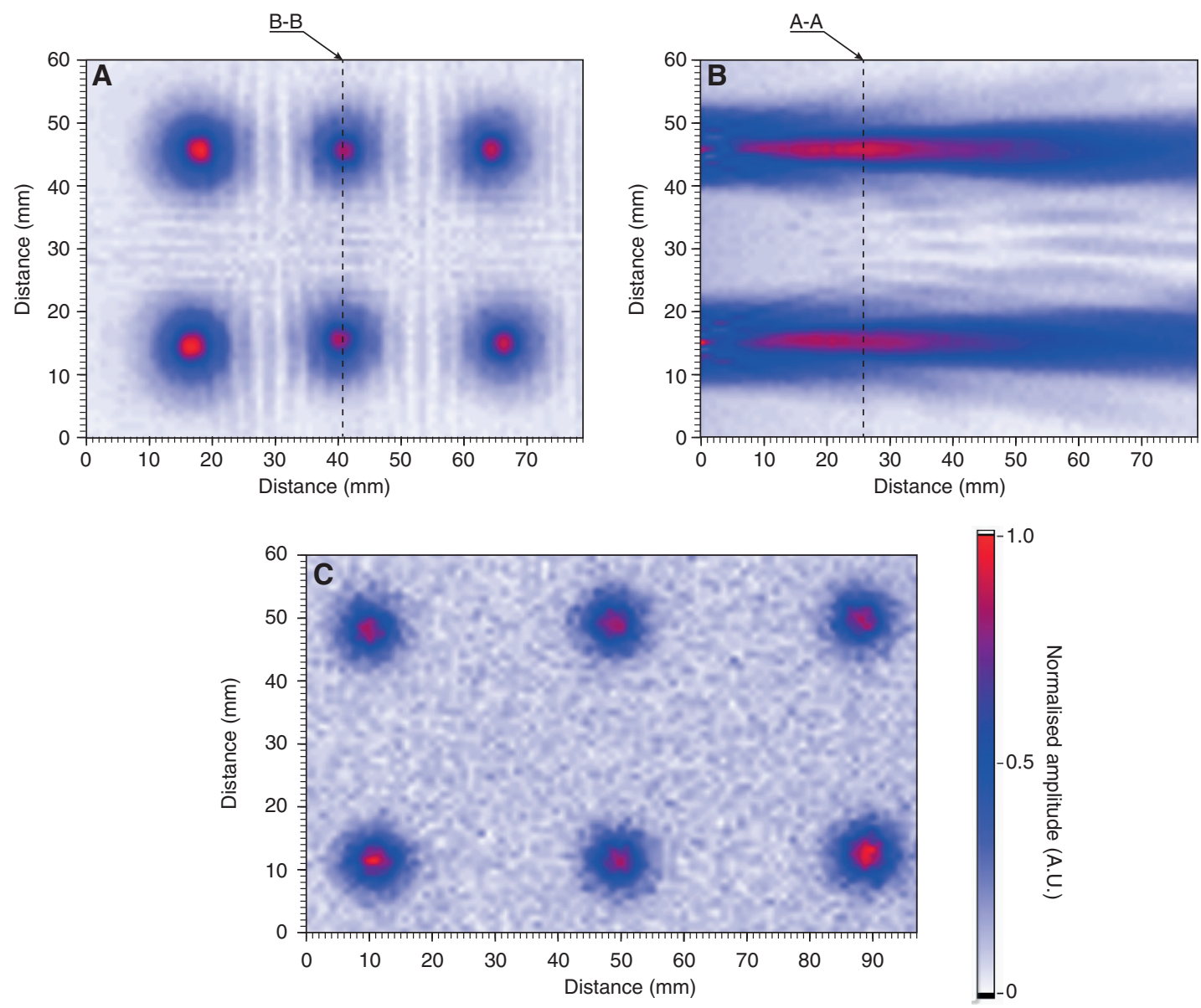

Figure 6 Field scans of plate sonicators. Scans A and B are the $x y$ and $x z$ scans of the OptiCell ${ }^{\mathrm{TM}}$ sonicator. Line B-B indicates the location of scan B, wheras line A-A indicates the location of scan A. Scan C is the is the $x y$ scan of the 24 -well plate sonicator. All scans are at the same scale. Minor side lobes are evident in the OptiCell ${ }^{\mathrm{TM}}$ sonicator due to the close proximity of the transducers. This was not seen in the $24-w e l l$ plate sonicator.

and B shows the mean beam profiles in the $x$ and $y$ axis at the acoustic focus. Figure $7 \mathrm{C}$ shows a whisker plot of the beam area at the acoustic focus using a $-3-\mathrm{dB}$ cutoff. The mean focal area for device A was $148 \pm 21 \mathrm{~mm}^{2}$. In comparison, the mean focal area for device B was $106 \pm 11 \mathrm{~mm}^{2}$.

The acoustic pressures generated by each element for both devices are shown in Figure 8A. The mean maximum peak-peak acoustic pressure generated by each transducer was 1.5 MPa. Between transducers in each device, the coefficient of variance was $6 \%$, and between the mean of each device, $3 \%$. The PZ26 elements have a variance of $\approx 8 \%$ from the manufacturer; hence, any variation in acoustic output of the completed device below $10 \%$ can be considered reasonable. Commercial therapeutic ultrasound devices have shown to have an acoustic output variance of $60 \%$ [10]; hence, we believe our devices have an acceptable variance.
When mounting the PZT elements into the case with the glass bubble/epoxy backing, impedance measurements showed a large reduction in modal interference in addition to a resonance frequency increase. The impedance of the electrical and mechanical resonance was increased and decreased, respectively. This indicated the glass bubble/epoxy was damping the mechanical oscillations. Nevertheless, this damping resulted in a smaller electrical impedance variance between devices. Figure 8B shows the benefits of electrical impedance matching. Without impedance matching, the output followed an inverse slope of 26.9, whereas with impedance matching, the output followed an inverse slope of 8.55 . Hence, impedance matching increased the pressure output by a factor of 3.15. Looking at the non-linearity ratio (Figure 8C), it is evident that as the acoustic pressure increases, so does the nonlinearity, thus limiting the maximum acoustic pressure possible for operation in the linear regime. The acoustic 

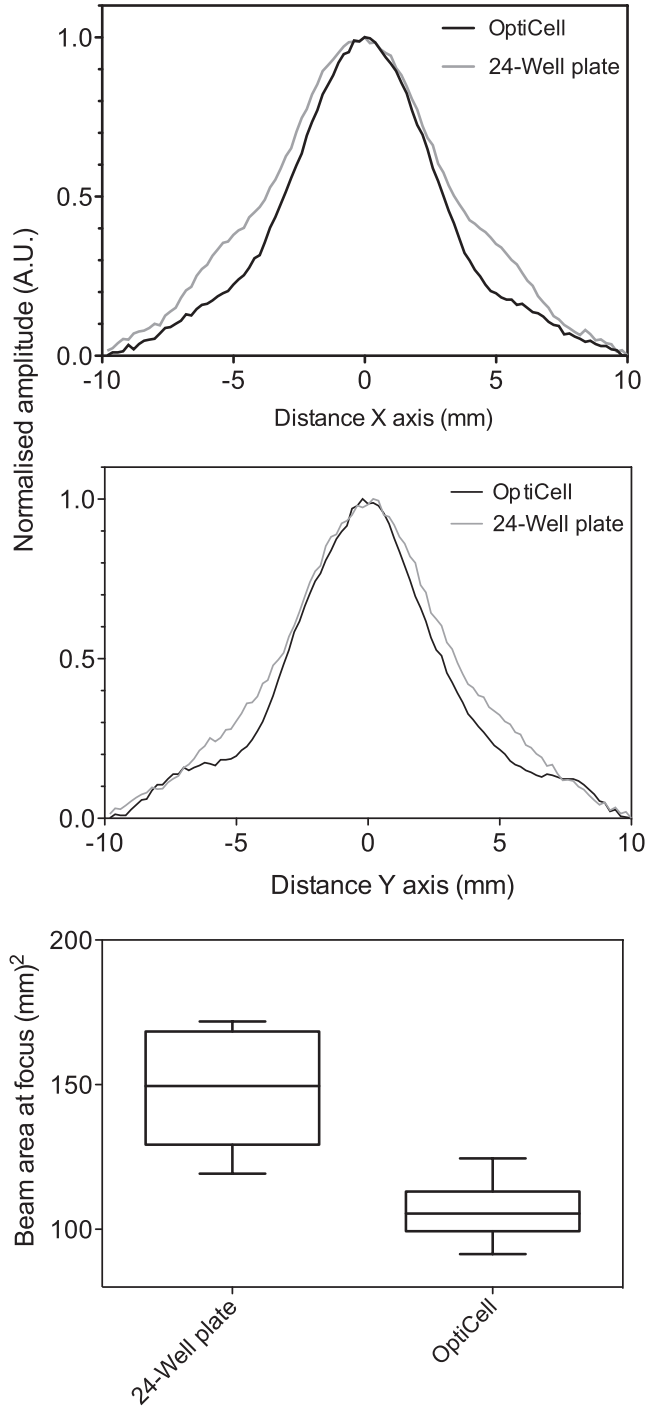

Figure 7 Beam profile scans at acoustic focus for both devices (A and B) and whisker plot of the focal area for the two devices using a -3-dB cutoff. A statistically significantly smaller beam area is evident in the OptiCell ${ }^{\mathrm{TM}}$ sonicator, due to the close proximity of the transducers (cf. Figure 6).

pressure and nonlinearity were statistically significantly similar between devices ( $\mathrm{p}=0.95$ and $\mathrm{p}=0.80$, respectively).

When driving the devices at $100 \mathrm{~W}$ RMS, device A reflected $2 \mathrm{~W}$, whereas device $\mathrm{B}$ reflected $5 \mathrm{~W}$, i.e., an electrical power reflection of $2 \%$ and $5 \%$, respectively.

As these devices are targeted to be used as templates for low-cost scientific ultrasound treatment, Table 2 shows the total cost of consumables and time used to fabricate a single device (rounded up). Total material cost of each custom made device was $€ 220$. The total work time required was $8 \mathrm{~h}$.

Figure 9 shows a photograph of the completed devices.

\section{Operation}

Each device was controlled by a custom program written in LabVIEW (National Instruments Corporation, Austin, TX, USA). The program was based on a "one-button" operation principle. It only contained two buttons: read file and start. Prior to initiation of the experimental work, the user is required to write a file indicating the name, frequency, pressure, intensity, and duration required for each experiment. This file is read by the software and all parameters are automatically calculated. The user is only required to follow the on-screen instructions and press "OK" to proceed. Figure 9C shows a screenshot of the software in operation. The software was presented to several independent cellular biology researchers to evaluate its ease of use. All users were able to use the program effectively with no training.

\section{Discussion}

\section{Electrical impedance}

Using transmission line transformers to reduce electrical reflection is effective and very cost and time efficient. Nevertheless, it is important to ensure that the cores chosen can adequately handle the magnetic flux generated. Larger magnetic flux results in higher temperatures, which in consequence reduces the magnetic permeability. The small cores used in this case saturated after $5 \mathrm{~min}$ when driven at $100 \mathrm{~W}$ with no additional cooling. When saturated, the transformers reflected up to $95 \%$ of the incident power. A solution to saturation is to maintain a sufficiently low temperature by cooling them or to use larger cores and thicker gauge wires.

Furthermore, impedance matching can also be performed for the third and fifth harmonics, allowing sonication over a broad range of frequencies.

\section{Acoustic output}

Using a hard PZT, we achieved a narrow bandwidth with a high acoustic output. Nevertheless, this template allows for any type of element to be used, i.e., adjustable to the end users requirements. To achieve a larger bandwidth, a softer element, with higher internal damping, or a denser backing could be used. If higher acoustic outputs are desired, focused elements may be used. It is important 

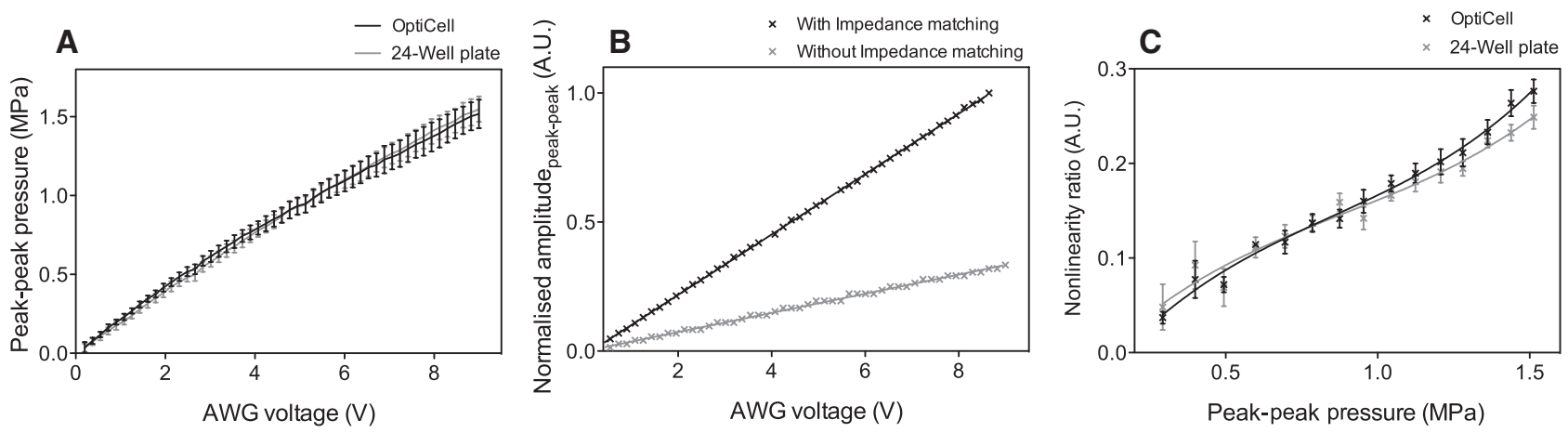

Figure 8 Acoustic output from sonicators. (A) Mean peak-to-peak acoustic pressure as a function of arbitrary waveform generator (AWG) voltage. The acoustic pressure output is not linear in relation to the voltage input. (B) Normalised mean peak-to-peak acoustic pressure as a function of AWG voltage with and without impedance matching. Electrical impedance matching effectively tripled the acoustic output. (C) Mean non-linearity ratio a function of mean peak-to-peak acoustic pressure. As acoustic pressure increases, so does the nonlinearity explaining the nonlinear relationship between input voltage and output pressure. Error bars indicate standard deviations.

Table 2 Time and cost for manufacturing and characterising one multi-element ultrasound treatment device.

\begin{tabular}{lrr}
\hline Process & $\begin{array}{r}\text { Consumable } \\
\text { cost }(\boldsymbol{\ell})\end{array}$ & $\begin{array}{r}\text { Duration } \\
\text { (working hours) }\end{array}$ \\
\hline 3D print and post-processing print & 120 & 2 \\
Ultrasound transducer fabrication & 50 & 1 \\
Impedance matching & 25 & 2 \\
Electronics connectors and cables & 25 & - \\
Field characterisation & - & 3 \\
Total & 220 & 8 \\
\hline
\end{tabular}

A total cost of $€ 220$ in consumables and 8 work hours was required to build and calibrate each device. The major component cost was the $3 \mathrm{D}$ print material.

to note that much higher acoustic pressures result in increasingly nonlinear sound fields and smaller focal areas. Depending on the application, it may be critical to control the amount of nonlinearity, e.g., to avoid shockwave formation or inertial cavitation.

\section{Practical considerations}

When using the devices, acoustic attenuation needs to be minimised. Hence, when using device A, a self-adhering thin membrane (TopSeal ${ }^{\mathrm{TM}}$-A, Perkin Elmer) was placed on the upper surface of the plate and the plate was placed in the device inverted. The thin membrane reduced the output by $<1 \%$; hence, its effects can be considered negligible.

The CAD files and LabVIEW program are available as supplementary data and on our website (org.uib.no/ microacoustics). The free LabVIEW Run-Time Engine is required to run the executable (.exe) file.

\section{Cost}

Manufacturing such a device in-house helps drastically reduce the cost associated with such expensive and dedicated setups. A typical underwater ultrasound transducer costs in the range of $€ 600-1000$, whereas this device costs close to a third of that value, with an additional five transducers and is customised to the experimental need.

\section{User notes and recommendations}

Prior to assembling the base plate to the extension frame, it is recommended that the base plate, with the embedded transducers, is calibrated in a water tank. Calibrating without the extension frame helps prevent accidental hydrophone contact and damage. All the transducers should be driven simultaneously. The device should be calibrated in the following order:

1. Evaluate bandwidth and centre frequency using pulse-receive technique as described in Acoustic field characterisations' section.

2. Drive transducer at low power at centre frequency. Position hydrophone at equivalent plate/OptiCell ${ }^{\mathrm{TM}}$ level. Calibrate for a range of voltages. Perform same calibration for all other transducers.

3. Check the electrical impedance and your reflection coefficient (e.g., $\pm 1 \mathrm{MHz}$ of your centre frequency). 


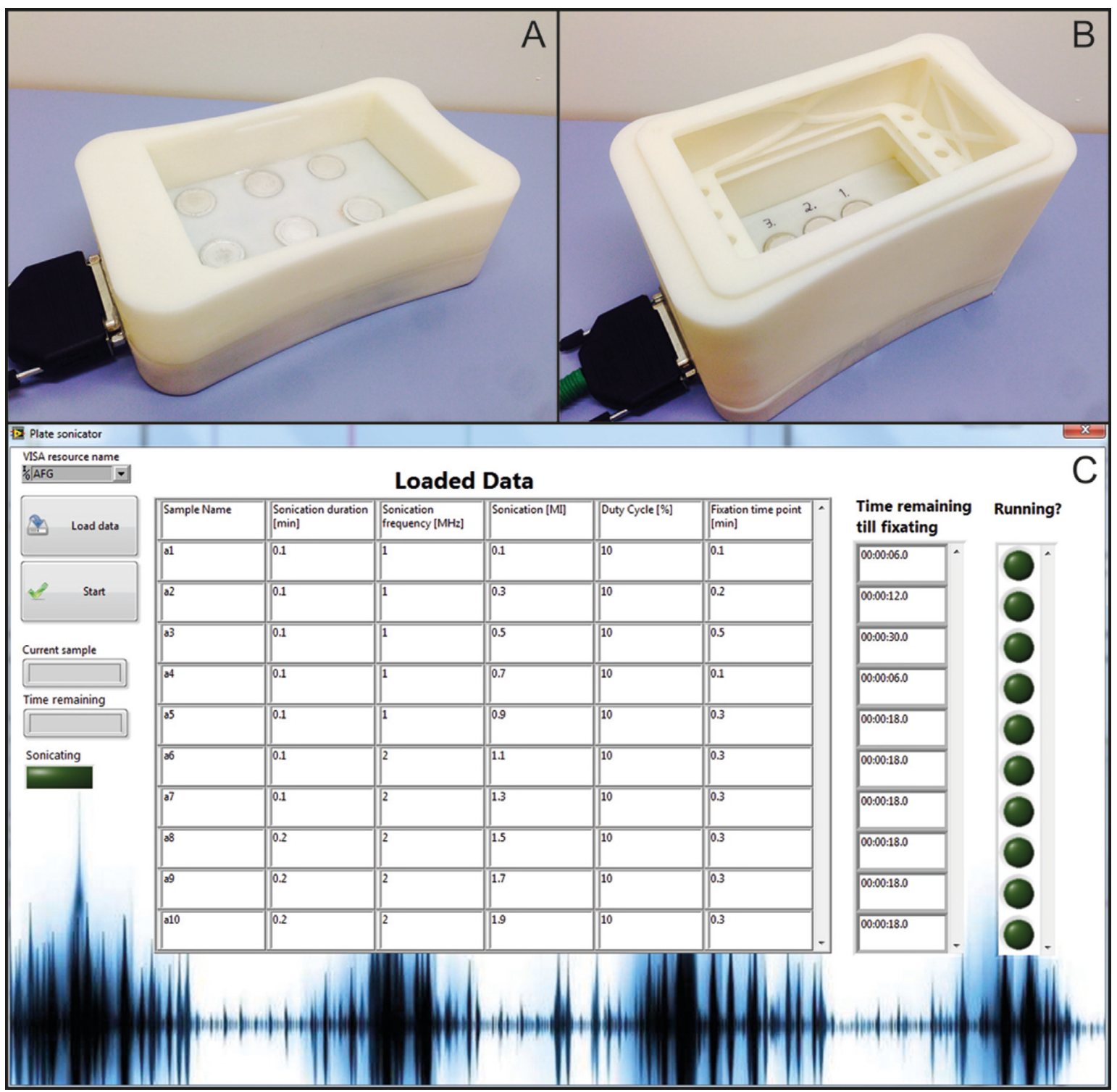

Figure 9 Completed devices and software. Photograph of the completed 24-well plate sonicator (A) and OptiCell ${ }^{\mathrm{TM}}$ (B). (C) Screen capture of the user software interface.

The acoustic pressures should be very similar between all devices $( \pm 5 \%)$. If the difference is substantially higher or lower, this will be seen in the electrical impedance scans during transducer assembly; hence, this issue can be prevented. Typical problems are bad electrical connections, e.g., damaged silver paint, poor soldering, or shortcircuiting. If a transducer is working poorly, and none of these typical problems seem to be at fault, it may be due to element damage. In this circumstance, the transducer should be replaced. Prior to each use, we recommend testing the device by running for a few seconds and checking (1) the power forward and reflected and (2) filling the chamber to the plate/OptiCell ${ }^{\mathrm{TM}}$ with degassed water, driving the elements at a moderate pressure, e.g., $\mathrm{MI}=0.3$ at
$20 \%$ duty cycle, and looking for small peaks at the water/ air interface. These should be the same height for all transducers. A lower height may indicate a faulty transducer.

\section{Conclusion}

In this paper, we have described the procedure to build, assemble, and characterise multi-element biological cell sonication treatment chambers. These chambers have high electrical efficiency, i.e., electrical reflections of $<10 \%$, by taking advantage of transmission line transformers. These devices are easy to construct, i.e., no special tools are required, and can be produced in bulk. There is a low 
variance between devices, independent of slight variances in construction, e.g., variable density of the backing material and different transformer design. The acoustic outputs between all 12 transducers had a variance of $6 \%$. In addition, complete fabrication and characterisation can be complete within 5 working days, taking into account epoxy curing times, at a low cost of €220. These ultrasound treatment chambers provide a template for multiple variations of ultrasound based experimental setups. These chambers have shown to be extremely easy to use and improve the experimental throughput in biology and ultrasound-related experiments. We also provide the CAD and LabVIEW files for building and operating the devices.

Acknowledgments: The study was supported by MedViz, a research cluster of groups from Haukeland University Hospital, University of Bergen, and Christian Michelsen Research AS. We would also like to thank The Michelsen Centre for Industrial Measurement Science and Technology for supporting this research.

\section{References}

[1] ASTM International. ASTM standard E1065. West Conshocken, PA: ASTM International 2008.

[2] Awad SB, Nagarajan R. Ultrasonic cleaning. In: Kohli R, Mittal KL, editors. Developments in surface contamination and cleaning. Oxford: William Andrew Publishing 2010: 225-280.

[3] Delalande A, Bouakaz A, Renault G, et al. Ultrasound and microbubble-assisted gene delivery in Achilles tendons: long lasting gene expression and restoration of fibromodulin KO phenotype. J Control Rel 2011; 156: 223-230.

[4] Delalande A, Kotopoulis S, Rovers T, Pichon C, Postema M. Sonoporation at a low mechanical index. Bubble Sci Eng Technol 2011; 3: 3-11.

[5] Eisenmenger W. The mechanisms of stone fragmentation in ESWL. Ultrasound Med Biol 2001; 27: 683-693.

[6] Gourevich D, Hertzberg Y, Volovick A, et al. Ultrasound-mediated targeted drug delivery generated by multifocal beam patterns: an in vitro study. Ultrasound Med Biol 2013; 39: 507-514.

[7] Hu Y, Wan JM, Yu AC. Cytomechanical perturbations during low-intensity ultrasound pulsing. Ultrasound Med Biol 2014; 40: 1587-1598.

[8] International Electrotechnical Commission. Ultrasonics - hydrophones - part 2: calibration for ultrasonic fields up to $40 \mathrm{MHz}$. Geneva, Switzerland: International Electrotechnical Commission 2013.

[9] Khanna A, Nelmes RT, Gougoulias N, Maffulli N, Gray J. The effects of LIPUS on soft-tissue healing: a review of literature. Br Med Bull 2009; 89: 169-182.
[10] Kollmann C, Vacariu G, Schuhfried O, Fialka-Moser V, Bergmann $\mathrm{H}$. Variations in the output power and surface heating effects of transducers in therapeutic ultrasound. Arch Phys Med Rehab 2005; 86: 1318-1324.

[11] Kopechek JA, Kim H, McPherson DD, Holland CK. Calibration of the 1-MHz Sonitron ultrasound system. Ultrasound Med Biol 2010; 36: 1762-1766.

[12] Kotopoulis S, Wang H, Cochran S, Postema M. Lithium niobate ultrasound transducers for MRI-guided ultrasonic microsurgery. IEEE Trans Ultrason Ferroelectr Freq Control 2011; 58 : 1570-1576.

[13] Kotopoulis S, Dimcevski G, Gilja OH, Hoem D, Postema M. Treatment of human pancreatic cancer using combined ultrasound, microbubbles, and gemcitabine: a clinical case study. Med Phys 2013; 40: 072902.

[14] Kujawska T, Secomski W, Bilmin K, Nowicki A, Grieb P. Impact of thermal effects induced by ultrasound on viability of rat C6 glioma cells. Ultrasonics 2014; 54: 1366-1372.

[15] Lau PC, Zheng SF, Ng WT, Yu SC. Inoperable pancreatic adenocarcinoma rendered complete remission by high-intensity focused ultrasound concurrent with gemcitabine-capecitabine chemotherapy: case report and topic review. J Dig Dis 2012; 13: 60-64.

[16] Lee I, Lo T-L, Young T-H, et al. Differentiation of neural stem/ progenitor cells using low-intensity ultrasound. Ultrasound Med Biol 2014; 40: 2195-2206.

[17] Nomikou N, Li YS, McHale AP. Ultrasound-enhanced drug dispersion through solid tumours and its possible role in aiding ultrasound-targeted cancer chemotherapy. Cancer Lett 2010; 288: 94-98.

[18] Olkku A, Leskinen JJ, Lammi MJ, Hynynen K, Mahonen A. Ultrasound-induced activation of Wnt signaling in human MG-63 osteoblastic cells. Bone 2010; 47: 320-330.

[19] Patil R, Bhoir P, Deshpande P, Wattamwar T, Shirude M, Chaskar P. Relevance of sonochemistry or ultrasound (US) as a proficient means for the synthesis of fused heterocycles. Ultrason Sonochem 2013; 20: 1327-1336.

[20] Polonschii C, Bratu D, Gheorghiu E. Appraisal of fish behaviour based on time series of fish positions issued by a 3D array of ultrasound transducers. Aquacult Eng 2013; 55: 37-45.

[21] Postema M. Fundamentals of medical ultrasonics. London: Spon Press 2011.

[22] Preston RC. Output measurement for medical ultrasound. New York: Springer-Verlag 1991.

[23] Rizzo LY, Theek B, Storm G, Kiessling F, Lammers T. Recent progress in nanomedicine: therapeutic, diagnostic and theranostic applications. Curr Opinion Biotechnol 2013; 24: 1159-1166.

[24] Sevick J. Transmission line transformers handbook. Costa Mesa, CA: Amidon Associates 1997.

[25] Sevick J. Transmission line transformers, 4th ed. Atlanta, GA: Noble Publishing Corporation 2001.

[26] Wei F, Gao GZ, Wang XF, et al. Quantitative determination of oil content in small quantity of oilseed rape by ultrasoundassisted extraction combined with gas chromatography. Ultrason Sonochem 2008; 15: 938-942. 